\title{
Evaluation of the Professional Motivation of Anaesthesiology Residents and Attendings in Portugal
}

\section{Euroanaesthesia}

\section{8}

Joana Pedreira' ', Camile Lanzaro', Luis Ferreira' ', Ana Bernardino', Maria Lurdes Bela'

1. Centro Hospitalar e Universitário de Coimbra

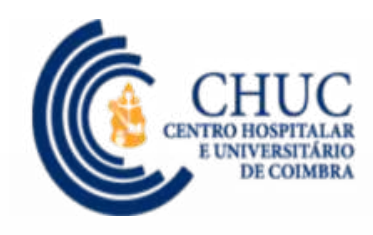

\section{BACKGROUND}

Professional motivation is fundamental for performance of good medical practices in all its aspects: human and social, technical and intellectual.

The goal of this study is to evaluate the professional motivation of Anaesthesiology residents and attendings in Portugal and to identify factors that may influence it.

\section{METHODS}

Study design

Observational descriptive analytical study

Target population

Anaesthesiologists professionals in Portugal

Data collection

Online survey sent by e-mail

October and November 2017

Variables

\section{Demographic data}

\section{Professional activity data}

Multi-Factor Scale of Working Motivation questionnaire (Multi-Moti):

5 point Likert scale (from 1 - "completely disagree" to 5 - "completely agree")

28 items organized in 4 dimensions: work organization (WO), performance motivation (PM), self-realization and professional progression (RP) and emotional involvement (EI)

Statistical analysis

SPSS $24.0^{\circledR}, a=0.05$

Descriptive: frequencies, percentages, mean and median

Analytical: T test, Mann-Whitney U, ANOVA and Spearman correlation

\section{RESULTS}

\begin{tabular}{|c|c|}
\hline 110 Physicians \\
\hline 56 residents $(50,9 \%)$ & 54 attendings $(49,1 \%)$ \\
\hline $\begin{array}{c}\text { Median age } 31 \text { years } \\
\text { (min } 26-\text { max 62) }\end{array}$ & $\begin{array}{c}\text { Attendings years of work } \\
\text { median } 9,5 \text { years }\end{array}$ \\
\hline
\end{tabular}

Sample characterization (table 1,2 and 3)

Table 1 . Demographic data

Variable $\quad \mathrm{N}(\%)$

Gender $\quad$ Female $80(72,7 \%)$
Male $30(27,3 \%)$

Children

$41(37,3 \%)$

Single $48(43,6 \%)$ Married $58(52,8 \%)$

Divorced $4(3,6 \%)$
Table 2 . Other data

\begin{tabular}{cc} 
Variable & $\mathbf{N}(\%)$ \\
\hline Medication & $28(25,5 \%)$ \\
$\begin{array}{c}\text { Chronic } \\
\text { disease }\end{array}$ & $17(15,5 \%)$ \\
Leisure & $91(82,7 \%)$ \\
\hline $\begin{array}{c}\text { Alcohol, } \\
\text { smoking, } \\
\text { drugs }\end{array}$ & $27(24,5 \%)$ \\
\hline
\end{tabular}

Table 3 . Professional activity data

\begin{tabular}{|c|c|c|c|}
\hline Variable & $\mathrm{N}(\%)$ & Variable & $\mathrm{N}(\%)$ \\
\hline Region & $\begin{array}{l}\text { North } 32(29,1 \%) \\
\text { Center } 48(43,6 \%) \\
\text { South } 28(25,5 \%)\end{array}$ & $\begin{array}{l}\text { Hospital } \\
\text { activity }\end{array}$ & $\begin{array}{l}\text { Public } 88(80,0 \%) \\
\text { Private } 2(1,8 \%) \\
\text { Both } 20(18,2 \%)\end{array}$ \\
\hline $\begin{array}{l}\text { Weekly } \\
\text { working } \\
\text { hours }\end{array}$ & $\begin{array}{c}<20 \text { h: } 1(0,9 \%) \\
20-40 \text { h: } 11(10,0 \%) \\
>40 \text { h: } 98(89,1 \%)\end{array}$ & $\begin{array}{c}\text { University } \\
\text { hospital }\end{array}$ & $67(60,9 \%)$ \\
\hline
\end{tabular}

Table 4. Professional motivation in the different domains of the Multi-Moti questionnaire in the total sample and between attendings and residents

\begin{tabular}{c|c|c|c|c|c|c|c|} 
& \multicolumn{2}{|c|}{ Total } & \multicolumn{2}{c}{ Aftendings } & \multicolumn{2}{c}{ Residents } & P \\
& $\begin{array}{c}\text { Mean } \\
\pm S D\end{array}$ & Median & $\begin{array}{c}\text { Mean } \\
\pm S D\end{array}$ & Median & $\begin{array}{c}\text { Mean } \\
\pm S D\end{array}$ & Median & value \\
\hline WO & $\begin{array}{c}3,02 \\
\pm 1,04\end{array}$ & 3,00 & $\begin{array}{c}3,23 \\
\pm 0,85\end{array}$ & 3,00 & $\begin{array}{c}2,80 \\
\pm 1,17\end{array}$ & 3,00 & $\mathbf{0 , 0 2 7}$ \\
\hline PM & $\begin{array}{c}3,65 \\
\pm 0,68\end{array}$ & 4,00 & $\begin{array}{c}3,73 \\
\pm 0,70\end{array}$ & 4,00 & $\begin{array}{c}3,54 \\
\pm 0,66\end{array}$ & 4,00 & 0,227 \\
\hline RP & $\begin{array}{c}3,85 \\
\pm 0,91\end{array}$ & 4,00 & $\begin{array}{c}3,93 \\
\pm 0,81\end{array}$ & 4,00 & $\begin{array}{c}3,78 \\
\pm 1,00\end{array}$ & 4,00 & 0,388 \\
\hline EI & - & 4,00 & - & 4,00 & - & 4,00 & 0,266 \\
\hline
\end{tabular}

Table 5 . Correlated variables with professional motivation

\begin{tabular}{|c|c|c|c|c|}
\hline Variable & Dimension & Single & Married & $\mathbf{p}$ \\
\hline \multirow[t]{2}{*}{$\begin{array}{c}\text { Marital } \\
\text { status }\end{array}$} & Wo & $3,33 \pm 0,93$ & $2,81 \pm 1,08$ & 0,027 \\
\hline & & Private & Both & $\mathbf{p}$ \\
\hline \multirow{3}{*}{$\begin{array}{l}\text { Hospital } \\
\text { activity }\end{array}$} & wo & $3,08 \pm 0,93$ & $2,60 \pm 1,35$ & 0,039 \\
\hline & $\mathrm{RP}$ & $2,50 \pm 0,71$ & $4,15 \pm 0,99$ & 0,041 \\
\hline & & University & No university & $p$ \\
\hline Hospital & RP & $3,72 \pm 0,98$ & $4,07 \pm 0,74$ & 0,034 \\
\hline
\end{tabular}

\section{DISCUSSION AND CONCLUSION}

Currently, Portuguese Anaesthesiologists seem to be professionaly motivated.

The gender, to have or not to have children, region, weekly working hours and the progression in medical career do not influence the degree of professional motivation.

Single anesthesiologists who practice in non-university or in the private hospitals have a higher professional motivation. 